\title{
Shapes and Fissility of Highly Charged and Rapidly Rotating Levitated Liquid Drops
}

\author{
L. Liao and R. J. A. Hill" \\ School of Physics and Astronomy, University of Nottingham, Nottingham NG7 2RD, United Kingdom \\ (Received 12 November 2016; revised manuscript received 8 June 2017; published 15 September 2017)

\begin{abstract}
We use diamagnetic levitation to investigate the shapes and the stability of free electrically charged and spinning liquid drops of volume $\sim 1 \mathrm{ml}$. In addition to binary fission and Taylor cone-jet fission modes observed at low and high charge density, respectively, we also observe an unusual mode which appears to be a hybrid of the two. Measurements of the angular momentum required to fission a charged drop show that nonrotating drops become unstable to fission at the amount of charge predicted by Lord Rayleigh. This result is in contrast to the observations of most previous experiments on fissioning charged drops, which
\end{abstract} \\ typically exhibit fission well below Rayleigh's limit.
}

DOI: 10.1103/PhysRevLett.119.114501

The liquid drop forms the basis of models of physics on an extraordinary range of length scales, from the astronomical scale down to the scale of the atomic nucleus. Plateau, in 1863, devised an experiment to study the shape stability of spinning astronomical objects, drawing parallels between the drop's surface tension and the self-gravitation of an astronomical body [1], a powerful analogy still used to gain insights in modern cosmology [2]. The successes of the nuclear liquid drop model are well known [3]. Its extension to include a centrifugal term has been used to illuminate ongoing studies of highly deformed, rapidly rotating heavy nuclei [3-7]. Drop models have also proven useful to understand the stability of charged molecular and metal clusters $[8,9]$.

The stable equilibrium shape of a rigidly rotating drop (analogous to the so-called "yrast" state of a rapidly spinning nucleus) is known to progress through a series of ellipsoidlike shapes with increasing angular momentum, transitioning from oblatelike to triaxial at a critical angular velocity [4]. Uncharged liquid drops continue to deform into a capsule-like shape and then a double-lobed, "dumbbell" shape, as the angular momentum is further increased, finally fissioning by scission of the dumbbell neck as equilibrium is lost [10]. A nonspinning, surface-charged free drop, on the other hand, remains spherical unless the charge exceeds a critical amount, whereupon it fissions by a "Coulomb explosion": a sharp peak forms on the surface, known as a Taylor cone, which emits a fine jet of microscopic daughter drops [11]. This process is employed in the atomization of liquids, forming the basis of electrospray mass spectrometry and nanoparticle deposition, electrostatic spraying, and many other important technologies

Published by the American Physical Society under the terms of the Creative Commons Attribution 4.0 International license. Further distribution of this work must maintain attribution to the author(s) and the published article's title, journal citation, and DOI.
$[12,13]$. Lord Rayleigh predicted that a charged drop should become unstable to fission when the "fissility parameter" $x=Q^{2} / 64 \pi^{2} \epsilon_{0} a^{3} T$, the ratio of the electrostatic energy of the drop to twice its surface energy, exceeds 1 ; here $Q, T$, and $a$ are the charge, surface tension, and radius of the spherical drop at rest, respectively $[3,14]$.

Here, we investigate the situation in which the drop is endowed with both significant surface charge and angular momentum. The question arises: by which of these modes does a spinning and charged drop fission? Is there an abrupt change from electrospraylike Taylor cone fission to dumbbell fission, or is there a gradual variation with charge and angular momentum? Here, we observe an unusual mode of fission which appears to be a hybrid of both fission modes.

Our study affords a novel way to test Rayleigh's theory. Most previous experiments on nonspinning drops observe fission at $x$ significantly less than $1[11,15-21]$. In contrast, our results indicate fission at $x=1.0$. A crucial difference between our experiments and previous ones is that we do not reach the critical charge by evaporating the drop. Instead, we induce fission by spinning the charged drop.

We employed diamagnetic levitation in order to work with cm-scale free drops [22-29]. Levitation was achieved using a custom-built $18.5 \mathrm{~T}$ superconducting magnet with a 58-mm diameter, room temperature vertical bore. The large bore (for such a magnet) is required so that image charges on the walls of the bore do not destabilize the levitation. Previously, Rhim et al. demonstrated the use of acoustically applied torque to fission charged drops in an electrostatic balance, but obtained limited quantitative data for highly charged drops [30].

We levitated $\sim 1 \mathrm{ml}$ drops of a mixture of tertbutanol and water (TBW), one at a time (Fig. 1). This liquid has a large enough magnetic susceptibility to be levitated in our magnet, has a relatively low surface tension, reducing the voltage required to fission the drop, and a relatively large viscosity to help dampen oscillations and keep the drop in rigid-body rotation. In addition to the TBW mixture, we 
(a)

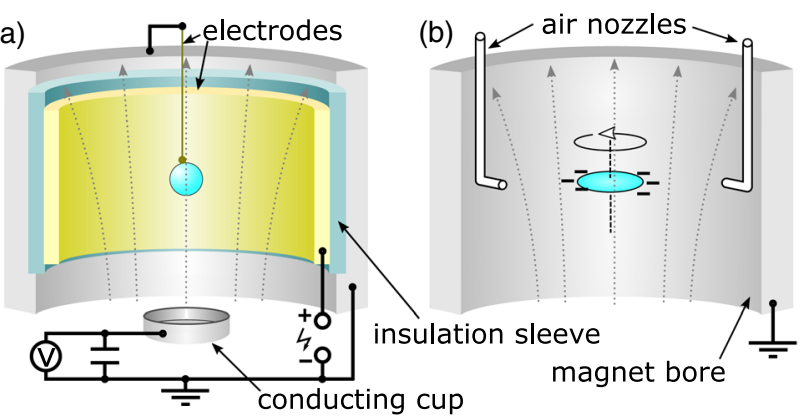

FIG. 1. Cutaway schematic drawings showing a drop levitating near the top of the $58 \mathrm{~mm}$-diameter magnet bore. Magnetic field lines are illustrated by dashed gray lines. (a) Configuration used to charge the drop, including the setup used to measure the charge on the drop. (b) Configuration used to spin a charged drop.

also studied drops of ethanol since there is less uncertainty in the values of the surface tension and density of this pure liquid, but at the expense of having a lower viscosity [31]. The drops are made almost weightless by the diamagnetic body forces counteracting gravity; stationary drops are spherical to a good approximation [27].

A drop was introduced to the stable levitation point in the magnetic field using a glass pipette. The volume of liquid expelled from the pipette was determined from the change in weight of the pipette. To charge the drop, a copper pipe electrode was first fitted inside the bore and connected to a stable high voltage power supply, applying up to $7 \mathrm{kV}$ to the pipe with respect to ground [Fig. 1(a)]. The tip of a thin, grounded wire was touched briefly to the drop, depositing a charge of up to $7 \mathrm{nC}$ on its surface, with uniform charge density.

Prior to the experiments on rotating drops, the charge imparted to nonrotating drops of varying volume was measured directly, in order to determine accurately the capacitance of the drop, grounding wire, pipe system as a function of drop radius. Once charged, the levitating drop was made to fall into a conducting cup in electrical contact with one plate of a $C=2 \mu \mathrm{F}$ calibrated capacitor, with opposite plate grounded, and the voltage $V_{C}=Q / C$ thus developed between the plates was measured with a fast, high impedance (10 G $\Omega$ ) voltmeter [Fig. 1(a)]. The drop was made to fall by lowering the magnetic field strength below that necessary for stable levitation. The charge imparted to drops by this method was found to be highly reproducible. Measuring the charge for various voltages on the copper pipe yielded the capacitance [31].

In subsequent experiments on rotating drops of TBW and ethanol, the drops were charged using exactly the same procedure, except that the charge was determined from the voltage on the pipe and the previously measured capacitance of the system. The electric field at the surface of these $\mathrm{cm}$-scale drops is always lower than the breakdown threshold of air prior to fission; charge is not lost by ionization. We measured the half life of the charge to be several hours, long on the time scale of the experiments, and independent of the air humidity. After charging a drop, the copper pipe was removed and two glass air nozzles inserted for spinning up the drop by airflow [Fig. 1(b)]. When air was supplied to the nozzles, the levitating drop began to rotate about a vertical axis coinciding with the axis of the magnet bore. The airflow was switched on gradually to avoid exciting large surface and center-of-mass oscillations of the drop. Weak excitations of $l=2,3$ and 4 spherical harmonics were occasionally observed, as in Ref. [26]. A second measurement of the drop volume was made after completing observations, to account for a small mass loss of up to $5 \%$ by evaporation during observation.

We first describe the observed shapes of uncharged, rotating drops, which have also been studied previously [26,28,37-41]. As the angular velocity $\Omega$ increased from rest (as measured by observing pollen grains added to the liquid), the equatorial radius of the drop expanded (Fig. 2, images a1-a2), until, at a particular $\Omega$, the drop lost its axisymmetric shape, elongating along an axis perpendicular to the rotation axis (image a3). While maintaining the airflow, i.e., continuing to apply a torque, the drop continued to elongate as its angular velocity fell, eventually developing a neck in the middle of the drop, giving the drop a dumbbell-like shape (images a4-a5). If we continued to apply airflow at this point, the drop

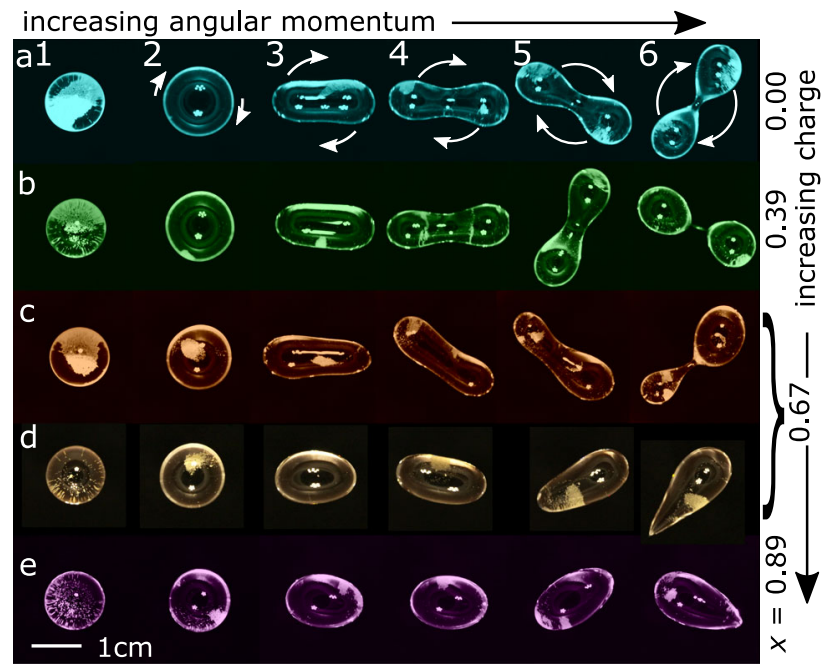

FIG. 2. The evolution of the equilibrium shapes of charged ethanol drops with increasing angular momentum; $x \propto(\text { charge })^{2}$ is the fissility. Drops (a)-(c): column 1 spherical, at rest; 2 oblatelike, close to the transition to a capsulelike shape; 3 capsule shaped; 4 double-lobed shape; 5 double lobed with pronounced neck ("dumbbell" shaped), prior to fission; 6 fission at the neck. Drop d (with the same $x$ as c) fissions by emitting a fine jet from a Taylor cone (see also enhanced image, Supplemental Material [31]). Drops with higher $x$ (e.g., drop e) fission by the Taylor cone mode. The fission of drop c appears to be a hybrid mode. The scale is the same in all images. See Supplemental Material for videos [31]. 
ultimately lost equilibrium by "necking": the neck thinned rapidly and then ruptured (image a6) causing the drop to fission into two daughter drops of equal volume, plus a third much smaller drop which is a remnant of the neck. These observations are in accordance with theory that predicts an axisymmetric, oblatelike stable equilibrium shape for a drop spun up from rest, below a critical angular velocity $\Omega_{\mathrm{II}}=\Omega_{\mathrm{II}}^{*} \sqrt{8 T /\left(\rho a^{3}\right)}$, where the dimensionless number $\Omega_{\mathrm{II}}^{*}=0.559$ was determined analytically by Chandrasekhar [10,42]; here, $\rho$ is the liquid's density. At this angular velocity, numerical modeling by Brown and Scriven and others has shown that a group of stable equilibrium shapes with lower symmetry bifurcates from the axisymmetric shape family [10,43-45]. This nonaxisymmetric so-called "two-lobed" shape family includes the capsulelike and dumbbell shapes observed here and elsewhere. The axisymmetric family loses stability at this angular velocity.

"Modestly" charged drops, $x<0.65$, behaved similarly to uncharged drops except that they lost axisymmetry at lower $\Omega$ with increasing $x$ (Fig. 2, images b1-b4). Above $x=0.68$, fission occurred by formation of a Taylor cone (e.g., Fig. 2, image e6). Fission occurred at progressively lower angular momentum with increasing $x$ above 0.68 . For $0.65<x<0.68$ we observed drops undergo fission by either Taylor cone fission or a double-lobed fission mode in which the volumes of the lobes were unequal. Fission in the latter case produced daughter drops of unequal volume (e.g., Fig. 2, image c6). Highly asymmetric double-lobed fission appeared similar in character to Taylor cone fission. Frequently, drops that ultimately fissioned from an asymmetric double-lobed shape developed a Taylor cone-like bump at one end (without fissioning) during spin-up, before the deformation spontaneously decayed and the drop went on to fission by necking.

To obtain quantitative comparisons with previous numerical and experimental work, we first measured the length of the longest axis (or axes) of the drop $R$ and its angular velocity $\Omega$ as the angular momentum of the drop was increased. The drop was spun up slowly to an angular momentum just short of that required for fission, and then allowed to spin down by cutting the airflow. In Fig. 3 we have plotted the dimensionless length $R^{*}=R / a$ against the dimensionless angular velocity $\Omega^{*}=\Omega \sqrt{\rho a^{3} /(8 T)}$ for a representative sample of charged and uncharged TBW drops. Arrows indicate the path taken with increasing and decreasing angular momentum. The angular velocity needed to cause the drop to break axisymmetry $\Omega_{\mathrm{II}}^{*}$ can be identified in this plot as the value of $\Omega^{*}$ where the gradient of the graph steepens sharply and becomes negative (a "backbend" [5]), beyond which $\Omega^{*}$ decreases with increasing angular momentum, owing to the rapidly increasing moment of inertia.

Comparing data from uncharged drops with Brown and Scriven's model (solid line) [10] shows that our measured

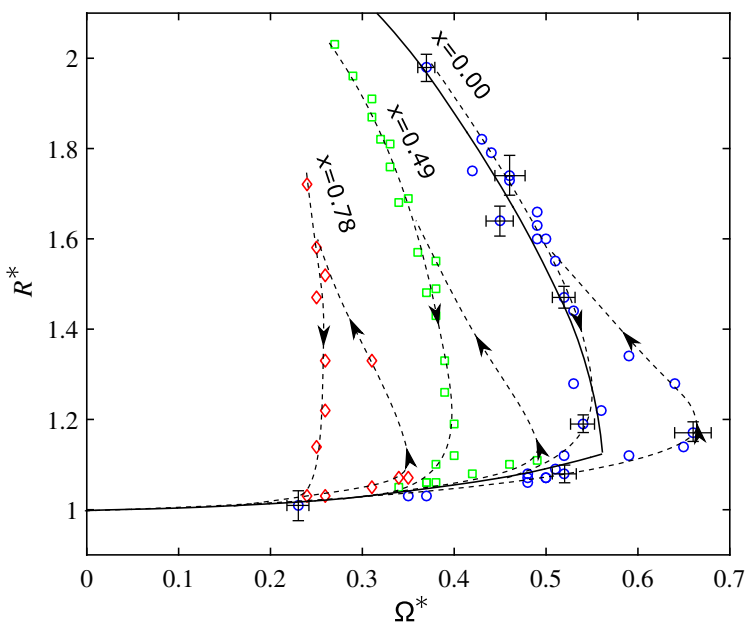

FIG. 3. Dimensionless length of the longest axis of the drop $R^{*}$ versus dimensionless angular velocity $\Omega^{*}$, shown for a representative set of charged and uncharged TBW drops. The fissility

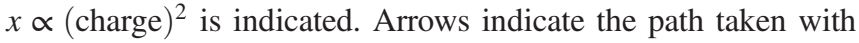
increasing and decreasing angular momentum. Solid line: numerical result for $x=0$, from Ref. [10]. Dashed lines are guides to the eye.

$\Omega_{\text {II }}^{*}$ on spin-up exceeds the theoretical value of 0.559 . Conversely, on spin-down, the drop returns to an axisymmetric shape at $\Omega_{\mathrm{II}}^{*}<0.559$. A similar effect was noted in studies of uncharged drops on the Space Shuttle, by Wang et al., who attributed it to the drop departing from equilibrium close to the two-lobed bifurcation point [40]. Taking the mean $(1 / 2)\left[\Omega_{\mathrm{II}}^{*}\right.$ (up) $+\Omega_{\mathrm{II}}^{*}($ down $\left.)\right]$ as a simple estimate, we obtain $\Omega_{\mathrm{II}}^{*}=0.57 \pm 0.02$ for $\mathrm{TBW}$ and $0.59 \pm 0.02$ for ethanol drops, which is in reasonable agreement with Chandrasekhar's value. With increasing $x$, the $R^{*}\left(\Omega^{*}\right)$ curves shift to lower angular velocity, with a corresponding shift of the measured values of $\Omega_{\mathrm{II}}^{*}$.

We used the measured values of $\Omega_{\mathrm{II}}^{*}$ to calculate the (dimensionless) angular momentum at the point where the axisymmetric shapes lose stability to the two-lobed family of shapes, $L_{\mathrm{II}}^{*}=I^{*} \Omega_{\mathrm{II}}^{*}$; here, $I^{*}$ is the (dimensionless) moment of inertia of the axisymmetric drop immediately prior to breaking axisymmetry. A good estimate of $I^{*}$ was obtained by approximating the axisymmetric shape as an oblate spheroid [31]. Also plotted on Fig. 4 is the angular momentum, $L_{\mathrm{II}}^{* \max }$, measured at drop fission. These data were obtained from the experimentally measured angular velocity and moment of inertia immediately prior to fission. In this case, the moment of inertia was estimated by modeling the shape as a uniform rod of length $2 R$.

Our measured values of $L_{\mathrm{II}}^{*}$, which decrease continuously with increasing $x$, from $L_{\mathrm{II}}^{*}=0.65 \pm 0.04$ at $x=0$, to approximately 0.23 at $x=0.85$, agree reasonably well with the results of a recent numerical study of surfacecharged and rotating drops by García-Garrido, Fontelos and Kindelán [45] (GFK), and also with Brown and Scriven's value of $L_{\mathrm{II}}^{*}=0.628$ at $x=0[10,46]$; the dotted-dashed 


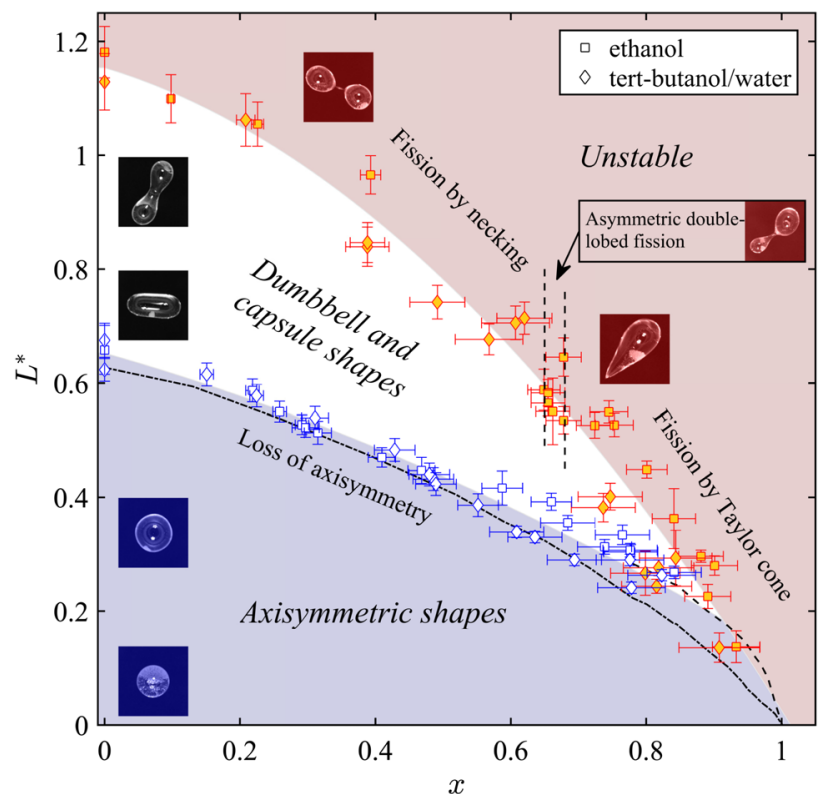

FIG. 4. Shape stability as a function of the fissility, $x \propto(\text { charge })^{2}$, and dimensionless angular momentum, $L^{*}$. Experimental data bound the lower region of stable axisymmetric shapes (shaded blue), and the region of stable dumbbell and capsule shapes (unshaded). Crossing into the upper region (shaded red), drops become unstable to fission by necking for $x<0.65$, or by formation of a Taylor cone $x>0.68$; for $0.65<x<0.68$, both Taylor cone and asymmetric dumbbell fission are observed. The boundaries of the shaded regions are quadratic fits to the data. Numerically determined boundaries for the axisymmetric shapes are indicated by the broken line for a free drop (dot-dash), and levitated drop (dashed).

line in Fig. 4 shows the results of our numerical model that closely reproduces the results of the equivalent GFK model [31]. Despite the crude estimate of the moment of inertia at fission, our finding that $L_{\mathrm{II}}^{* \max } \approx 1.15$ at $x=0$ is close to Brown and Scriven's theoretical value of 1.08 [10]. Above $x \approx 0.85$, it was not possible to observe whether there existed a stable nonaxisymmetric shape: drops fissioned by Taylor cone formation, apparently immediately after breaking axisymmetry.

The fission data $L_{\mathrm{II}}^{* \max }(x)$ clearly converge on a value of $x=1.0$ as $L^{*} \rightarrow 0:$ a quadratic fit passes directly through $x=1.0$ at $L^{*}=0$ (Fig. 4) [47]. The experimentally determined limit of stability of the axisymmetric shapes, $L_{\mathrm{II}}^{*}(x)$, is consistent with our calculated curve that includes the weak restoring forces necessary for levitation [27] (dashed line, Fig. 4), which passes through $x=1.0$ at $L^{*}=0$ [31]. Hence, we conclude that fission of a nonrotating $\mathrm{cm}$-scale free drop occurs at the charge predicted by Rayleigh, i.e., at $x=1$. In contrast, most recent experiments that study micrometer-sized drops using either an electrodynamic levitator or by studying electrosprays, measured values of $x$ substantially less than 1 at fission [11,15-20], including values that depend on the liquid [21].
This poses the question of whether or not fission occurs before Rayleigh's stability limit is reached in those experiments, by some hitherto unforeseen route. Duft et al. shed light on this question by showing unequivocally that fission of micrometer-sized ethylene glycol drops occurs concomitantly with loss of stability of the drop to a quadrupole deformation, in agreement with Rayleigh's theory [20]. Even in those experiments though, the apparent value of $x$ at fission, computed from the ostensible surface tension and the charge and volume of the drop, was $x \approx 0.7$ [20]. Duft et al. tentatively ascribed this result to increasing concentration of surface contaminants as the drop evaporated, altering the surface tension [20] (but, see Ref. [48]). As in all previous experiments to test Rayleigh's theory, a subcritically charged micrometer-sized drop of a volatile liquid was allowed to evaporate mass until the charge density became sufficiently high for it to undergo fission. In contrast, here, drops were made to undergo fission by spinning an initially subcritically charged drop, rupturing the drop by the combination of centripetal and electrostatic forces; evaporation was not necessary to obtain fission. We attempted to fission an isolated nonrotating drop by evaporation, as in previous studies, but the data from these experiments had a large scatter owing to the large uncertainty in the volume of the drop prior to fission. Attempts to charge a nonrotating drop to the Rayleigh limit, by applying the requisite charging voltage, caused the drop to fission well below the expected threshold charge, owing to the influence of the strong electric field at the tip of the grounding wire.

Our experiments show that reflection symmetry is spontaneously broken when the drop fissions by the Taylor cone mode: the drop forms a sharp peak at only one end of the drop. This is consistent with previous experiments (e.g., Ref. [11]) [49]. Unexpectedly, the stable equilibrium double-lobed shapes also break reflection symmetry where $x$ approaches the threshold for Taylor cone fission to be observed, leading to reflection asymmetric fission at higher angular momentum. Rhim et al. also observed similar asymmetric fission, without measurements of corresponding $x$ and $L$ values, using an electrostatic levitator [30]; this suggests that the mode is not an artifact of the particular method of levitation. Asymmetric double-lobed fission appears to be a hybrid mode, the smaller of the lobes becoming a stream resembling a Taylor cone-jet with increasing asymmetry. During spin-up to asymmetric double-lobed fission, drops develop a transient Taylor cone-like feature-frequently leading to Taylor cone fission at this point, terminating spin-updemonstrating that, between the abrupt appearance of Taylor cone fission at $x=0.65$, and the limit of doublelobed fission at $x=0.68$, Taylor cone and hybrid modes exist as alternative fission pathways.

There is currently considerable interest in reflection symmetry breaking in rotating nuclei, with recent 
experimental evidence [52]. Here, we observed a purely classical reflection symmetry-breaking mechanism in a charged rotating drop. We hope that this experimental result will spur further insights in this area, as have previous numerical studies of spinning liquid drops [4]. Through studying the stability and shapes of rotating charged droplets, we addressed the enduring puzzle of why droplets are observed to lose stability at charge seemingly at odds with Rayleigh's prediction. Our data provide unambiguous evidence that nonrotating $\mathrm{cm}$-sized droplets undergo fission at the charge given by Rayleigh's limit.

Acknowledgements.-The authors thank L. Eaves, K. A. Baldwin, E. B. Lindgren, E. N. Besley, and A. J. Stace for helpful discussions. This work was supported by the Engineering and Physical Sciences Research Council (Grant No. EP/I004599/1).

*Corresponding author richard.hill@nottingham.ac.uk

[1] J. A. F. Plateau, Annual Report of the Board of Regents of the Smithsionian Institution (1863), p. 270.

[2] V. Cardoso, O. K. C. Dias, and L. Gualtieri, Int. J. Mod. Phys. D 17, 505 (2008).

[3] H. J. Krappe and K. Pomorski, Theory of Nuclear Fission (Springer, New York, 2012).

[4] S. Cohen, F. Plasil, and W. J. Swiatecki, Ann. Phys. (N.Y.) 82, 557 (1974).

[5] A. Bohr, Rev. Mod. Phys. 48, 365 (1976).

[6] G. Anderson, S. E. Larsson, G. Leander et al., Nucl. Phys. A268, 205 (1976).

[7] A. Kardan, I. Ragnarsson, H. Miri-Hakimabad, and L. Rafat-Motevali, Phys. Rev. C 86, 014309 (2012).

[8] W. A. Saunders, Phys. Rev. Lett. 64, 3046 (1990).

[9] I. Last, Y. Levy, and J. Jortner, Proc. Natl. Acad. Sci. U.S.A. 99, 9107 (2002).

[10] R. A. Brown and L. E. Scriven, Proc. R. Soc. A 371, 331 (1980).

[11] A. Gomez and K. Tang, Phys. Fluids 6, 404 (1994).

[12] A. G. Bailey, Electrostatic Spraying of Liquids (Wiley, New York, 1988).

[13] J. B. Fenn, M. Mann, C. Meng, S. F. Wong, and C. Whitehouse, Science 246, 64 (1989).

[14] L. Rayleigh, Philos. Mag. 14, 184 (1882).

[15] C. B. Richardson, A. L. Pigg, and R. L. Hightower, Proc. R. Soc. A 422, 319 (1989).

[16] D. C. Taflin, T. L. Ward, and E. J. Davis, Langmuir 5, 376 (1989).

[17] E. J. Davis and M. A. Bridges, J. Aerosol Sci. 25, 1179 (1994).

[18] J. F. Widmann, C. L. Aardahl, and E. J. Davis, Aerosol Sci. Technol. 27, 636 (1997).

[19] K. Tang and R. D. Smith, J. Am. Soc. Mass Spectrom. 12, 343 (2001).

[20] D. Duft, H. Lebius, B. A. Huber, C. Guet, and T. Leisner, Phys. Rev. Lett. 89, 084503 (2002).

[21] J. N. Smith, R. C. Flagan, and J. L. Beauchamp, J. Phys. Chem. A 106, 9957 (2002).
[22] E. Beaugnon and R. Tournier, Nature (London) 349, 470 (1991).

[23] M. A. Weilert, D. L. Whitaker, H. J. Maris, and G. M. Seidel, Phys. Rev. Lett. 77, 4840 (1996).

[24] M. V. Berry and A. K. Geim, Eur. J. Phys. 18, 307 (1997).

[25] M. D. Simon and A. K. Geim, J. Appl. Phys. 87, 6200 (2000).

[26] R. J. A. Hill and L. Eaves, Phys. Rev. Lett. 101, 234501 (2008).

[27] R. J. A. Hill and L. Eaves, Phys. Rev. E 81, 056312 (2010).

[28] K. A. Baldwin, S. L. Butler, and R. J. A. Hill, Sci. Rep. 5, 7660 (2015).

[29] E. Beaugnon, D. Fabregue, D. Billy, J. Nappa, and R. Tournier, Physica B (Amsterdam) 294-295B, 715 (2001).

[30] W. K. Rhim, S. K. Chung, and D. D. Elleman, AIP Conf. Proc. 197, 91 (1990).

[31] See Supplemental Material at http://link.aps.org/ supplemental/10.1103/PhysRevLett.119.114501 for additional details, which includes Refs. [32-36].

[32] P. K. Kipkemboi and A. J. Easteal, Can. J. Chem. 72, 1937 (1994).

[33] J. Gliński, G. Chavepeyer, and J.-K. Platten, J. Chem. Phys. 102, 2113 (1995).

[34] F. A. M. M. Goncalves, A. R. Trindade, C. S. M. F. Costa, J. C. S. Bernardo, I. Johnson, I. M. A. Fonseca, and A. G. M. Ferreira, J. Chem. Thermodyn. 42, 1039 (2010).

[35] S. N. Makarov, G. M. Noetscher, and A. Nazarian, Low-frequency electromagnetic modeling for electrical and biological systems using MATLAB (Wiley, New York, 2016).

[36] P.-O. Persson and G. Strang, SIAM Rev. 46, 329 (2004).

[37] T. G. Wang, E. H. Trinh, A. P. Croonquist, and D. D. Elleman, Phys. Rev. Lett. 56, 452 (1986).

[38] E. H. Trinh and C. J.Hsu, J. Acoust. Soc. Am. 79, 1335 (1986).

[39] A. Biswas, E. W. Leung, and E. H. Trinh, J. Acoust. Soc. Am. 90, 1502 (1991).

[40] T. G. Wang, A. V. Anilkumar, C. P. Lee, and K. C. Lin, J. Fluid Mech. 276, 389 (1994).

[41] K. Ohsaka and E. H. Trinh, Phys. Rev. Lett. 84, 1700 (2000).

[42] S. Chandrasekhar, Proc. R. Soc. A 286, 1 (1965).

[43] R. A. Brown and L. E. Scriven, Phys. Rev. Lett. 45, 180 (1980).

[44] C.-J. Heine, IMA J. Numer. Anal. 26, 723 (2006).

[45] V. J. García-Garrido, M. A. Fontelos, and U. Kindelán, Q. J. Mech. Appl. Math. 66, 489 (2013).

[46] We follow Ref. [45] in our definition of $L^{*}$, which differs by a constant factor from that used in Ref. [10]. See Supplemental Material [31].

[47] The model cited in Ref. [45] shows a kink in the $L(x)$ fission curve at $x=0.8$; we cannot resolve this feature within the scatter of our data.

[48] K.-Y. Li, H. Tu, and A. K. Ray, Langmuir 21, 3786 (2005).

[49] Simultaneous fission from both ends was observed for drops in strong external electric fields [50,51].

[50] D. Duft, T. Achtzehn, R. Muller, B. A. Huber, and T. Leisner, Nature (London) 421, 128 (2003).

[51] R. T. Collins, K. Sambath, M. T. Harris, and O. A. Basaran, Proc. Natl. Acad. Sci. U.S.A. 110, 4905 (2013).

[52] L. P. Gaffney, P. A. Butler, M. Scheck, A. B. Hayes, F. Wenander, M. Albers, B. Bastin et al., Nature (London) 497, 199 (2013). 